\title{
Community-based wheelchair caster failures call for improvements in quality and increased frequency of preventative maintenance
}

\author{
Anand Mhatre $\mathbb{D}^{1 凶}$, Jon Pearlman ${ }^{1}$, Mark Schmeler ${ }^{1}$, Benjamin Krider ${ }^{1}$ and John Fried ${ }^{1}$ \\ (c) The Author(s) 2021
}

\begin{abstract}
STUDY DESIGN: Secondary data analysis of wheelchair failures and service repair logs from a network of wheelchair suppliers. OBJECTIVE: To determine the frequency of wheelchair caster failures and service repairs across wheelchair manufacturers and models and investigate the relationships between them.

SETTING: Wheelchair caster failures and service repairs occurred in the community.

METHODS: Reported caster failure types were classified based on the risk they pose for user injuries and wheelchair damage. Caster failures experienced by users of tilt-in-space and ultralightweight manual wheelchair models and Group 2, 3 and 4 power wheelchair models between January 2017 and October 2019 were analyzed using Chi-Square tests for independence. Correlational analysis of failures and service repairs was performed.

RESULTS: A total of 6470 failures and 151 service repairs reported across four manufacturers and five models were analyzed. Failure types were significantly associated with manufacturers and models, respectively. Users of tilt-in-space wheelchairs, who require greater seating support, experienced twice the proportion of high-risk caster failures than the ultralightweight manual wheelchair users. Similarly, Group 3 and 4 power wheelchair users, who have complex rehabilitation needs, experienced 15-36\% more high-risk failures than Group 2 users. Service repairs negatively correlated with high-risk manual wheelchair caster failures.

CONCLUSIONS: Wheelchair users who have greater seating and complex rehabilitation needs are at a higher risk for sustaining injuries and secondary health complications due to frequent caster failures. The study findings call for significant reforms in product quality and preventative maintenance practices that can reduce wheelchair failures and user consequences.
\end{abstract}

Spinal Cord (2022) 60:58-62; https://doi.org/10.1038/s41393-021-00689-3

\section{INTRODUCTION}

Wheelchairs are the primary means of mobility and independence for nearly $70 \%$ of people with spinal cord injuries in the United States $[1,2]$. However, these assistive devices do not yet meet the user needs fully. The growing research evidence on field evaluation of wheelchairs and laboratory-based testing has shown that manual and power wheelchairs suffer frequent failures [3-5]. Nearly 45-63\% wheelchairs in use experience one or more failures and/or repairs in a 6-month period and one-third of the failures result in adverse user consequences, including injuries and bruises $[4,6,7]$. Among wheelchair components, front caster failures account for $27 \%$ of all failures [8]. These failures can be risky. For example, caster wheel fractures can cause the wheelchair to tip and the user to fall out of the wheelchair and get hospitalized [4, 8, 9]. Evidence suggests that a user has to wait for an extended period of time for a repair. For example, based on a recent report from the Veterans Health Administration, about $40 \%$ of wheelchair repairs took more than a month for completion, during which veterans suffered physical and financial hardships [10]. The report cited a case in which a caster repair required 210 days. Without a functional wheelchair and longer repair times, the user loses access to work, school, and the community and may have to stay in bed. Failures are associated with pressure injuries, hospitalization and reduction in self-perceived health [11]. Consequently, it follows that failures negatively impact wheelchair user's health and can lead to personal and public health burdens.

Community studies have found that the incidence of wheelchair accidents reduces when preventative maintenance-related service repairs or active checkups are conducted $[12,13]$. If maintenance is not performed, a wheelchair user is ten times more likely to have had suffered a wheelchair accident in the past three years [14]. The Centers for Medicare and Medicaid Services (CMS) and private healthcare insurance pays for the repair and replacement of the wheelchair device but do not cover service repairs [15]. The CMS considers general maintenance to be the responsibility of the wheelchair user who usually lacks knowledge, training, tools, ability to repair, and access to replacement parts necessary for maintenance [16]. As a result, service repairs essential for preventative maintenance rarely happen, and users are left vulnerable to wheelchair breakdowns, health consequences, and hardships.

Very few research studies have investigated wheelchair caster failures, the risk they present to a wheelchair user, and mitigation of risk by preventative maintenance $[8,13,17,18]$. More evidence on the type and variation of caster failures across different

${ }^{1}$ Department of Rehabilitation Science and Technology, University of Pittsburgh, Pittsburgh, PA, USA. ${ }^{凶}$ email: anand.mhatre@pitt.edu 
Table 1. Wheelchair model descriptions.

$\begin{array}{ll}\begin{array}{l}\text { Wheelchair Model } \\ \text { Manual wheelchairs }\end{array} & \text { Description } \\ \text { Tilt-in-space wheelchair } & \text { Wheelchairs typically prescribed for less-active users who need seating and positioning support } \\ \begin{array}{l}\text { Ultralightweight wheelchair } \\ \text { Power wheelchairs }\end{array} & \text { Wheelchairs typically prescribed for users with active lifestyle and outdoor mobility needs } \\ \text { Group } 2 & \text { Wheelchairs typically having at most one power seat function and used on mildly uneven terrain } \\ \text { Group } 3 & \text { Wheelchairs typically having multiple power seat functions and can navigate thresholds, curbs, and other obstacles } \\ \text { Group } 4 & \begin{array}{l}\text { Wheelchairs typically with all power seat functions and added maneuvering capabilities that are not needed for use } \\ \text { in the home }\end{array}\end{array}$

wheelchair models is needed. Understanding the frequency of failures that are risky for users can inform design, quality testing standards development, part selection, repair, and maintenance strategies, and reduce the incidence of wheelchair failures and health consequences. This study aims to perform secondary data analysis of community caster failures across manufacturers and models of wheelchairs reported in the Wheelchair Repair Registry (WRR) [19] and explore their relationships. The study also evaluates the differences in caster survivability between manufacturers and the effect of service repairs on caster failure among wheelchair models.

\section{METHODS}

\section{Description of the Wheelchair Repair Registry}

The WRR is a wheeled mobility device failure and repair registry developed by the Rehabilitation Engineering Research Center at the University of Pittsburgh from wheelchair repair claims. The claims were reported by repair technicians from a network of wheelchair suppliers using LaborTracker, a repair data collection software. Currently, the registry has over 60,000 repairs conducted on more than 5000 wheelchair devices from 25 manufacturers. The devices include $60 \%$ power wheelchairs, $35 \%$ manual wheelchairs and $5 \%$ scooters. The development and structure of the WRR and the description of repairs and failures are published elsewhere [19].

\section{Data selection and cleaning}

Wheelchair models in WRR were assigned Healthcare Common Procedure Coding System (HCPCS) codes found on respective wheelchair order forms [20]. The system of coverage codes like HCPCS is notably used in developed countries but omitted in this manuscript. Manual wheelchair models were named based on their feature or functionality. Power wheelchair models were assigned group numbers depending on the wheelchair configuration listed on wheelchair order forms. For each model, the number of casters (left and/or right) and failures were computed. Caster repairs and failures reported for all manual and power wheelchair manufacturers and their models beginning in January 2017 until October 2019 were selected for data analysis. The analyzed caster failure types were classified based on the associated risks of wheelchair user injury and damage to other wheelchair parts [18]. Caster wheel fracture and bent part were designated as high-risk failures while bearing failure and worn-out tire were designated as low-risk failures. Repairs related to adjustment and lubrication of caster parts were categorized as service repairs. These repairs are part of preventative maintenance [21] and were performed while repairing or replacing another part. Duplicate or missing ticket and failure entries were discarded. Models with 100 caster failures or greater in total were selected for analysis.

\section{Statistical data analysis}

Chi-square tests for independence were conducted to evaluate the relationship between failures and wheelchair models and between failures and manufacturers. Kaplan-Meier survival curves were fitted to high-risk failures (found with casters having purchase date information) and logrank tests were performed to evaluate differences in survival rates across models. Linear regression analysis was conducted to evaluate the association between service repairs for manual wheelchair models and high-risk failures. Significance was set at $p<0.05$ and statistical analyses were performed manually.

\section{RESULTS}

A total of 6470 caster failures and 151 service repairs associated with 4 manufacturers and 5 wheelchair models were analyzed. Table 1 includes descriptions of manual and power wheelchair models analyzed in this study. Table 2 shows the distribution of wheelchair caster failures. Manufacturer names are anonymized using $\mathrm{M \# .}$

For manufacturer $\mathrm{M} 2$, the failures were associated with both the manual wheelchair models, $X^{2}(3, N=704)=42.15, \quad p<0.05$. Similarly, for M4, failures were associated with Group 2, 3, and 4 power wheelchairs, $X^{2}(6, N=4098)=207.66, p<0.05$. Purchase dates were available for ultralightweight model of $M 2$ and Group 3 models of M2 and M4. Accordingly, survival analyses were carried out with these models. Comparing the proportion of casters surviving at any specific time, significant differences were found between Group 3 wheelchair casters of manufacturers M2 and M4 as shown in Fig. $1, X^{2}\left(1, N_{\mathrm{M} 2}=224, N_{\mathrm{M} 4}=199\right)=5.36, p<0.05$. Caster service repairs were negatively correlated with high-risk failures of $M 1, M 2$, and $M 3$ manual wheelchair casters $(F(1,2)=$ $47.75, p<0.05)$ with an $R^{2}$ of 0.96 .

\section{DISCUSSION}

The proportion of high- and low-risk caster failures are unique to each wheelchair manufacturer and model reported in the WRR. There are two important study findings. First, comparing the proportion of highrisk failures to total failures among manual wheelchairs, the tilt-inspace models encounter nearly twice the high-risk failures than their ultralightweight counterparts. Second, among power wheelchairs, the proportion of high-risk failures to total failures showed a steady rise from 15 to $36 \%$ with an increasing group number of the models. These trends suggest that users who require a higher level of seating support and have complex rehabilitation needs are at a greater risk of experiencing caster failures that can cause user injury and other adverse consequences. Users are at additional risk if they use wheelchairs from manufacturers like M4, whose casters break faster than $\mathrm{M} 2$ according to the survival curves. Additionally, the high-risk caster failures occur within 1-2 years of wheelchair use, a finding noted in previous community evaluation studies and a common outcome of wheelchair standard testing studies [3, 4, 22]. These study findings call for urgent improvements in caster quality. To raise the quality of casters, the International Society of Wheelchair Professionals (ISWP) and the University of Pittsburgh's Rehabilitation Engineering Research Center on standards development have developed a caster testing protocol that simulates community failures in the laboratory and screens low-quality casters [9]. This protocol is currently under development for publication as an international wheelchair standard [23]. Based on testing results and iterative evaluations during the design phase, manufacturers can gain feedback to improve caster quality. ISWP has partnered with several national and international manufacturers on caster testing and redesign projects.

Along with high quality, preventative maintenance reduces failures in the community $[13,14]$, as found in this study. The ultralightweight models were serviced more times than tilt-in-space (see Fig. 2), which could have contributed to a lower high-risk failure count with active 
Table 2. Distribution of caster failures across wheelchair manufacturers and models.

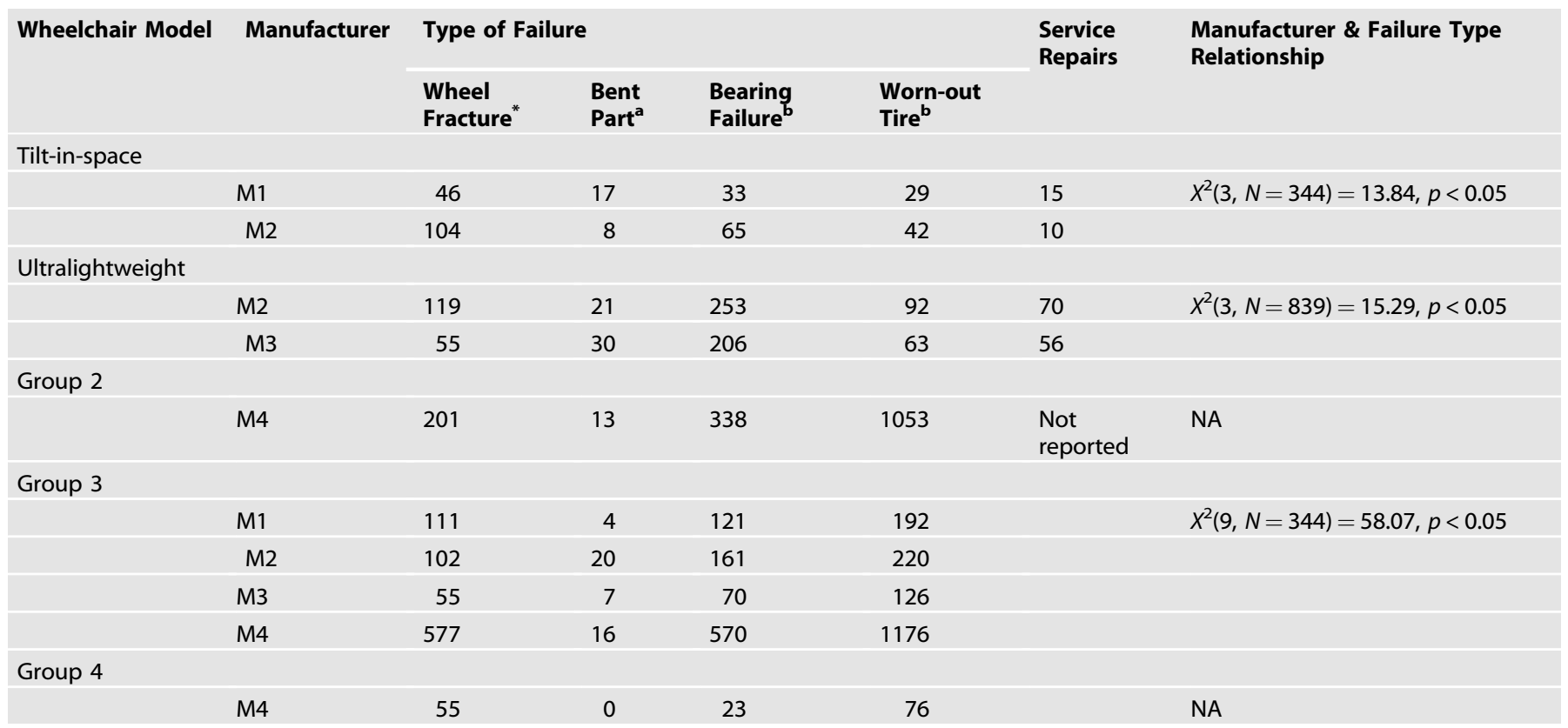

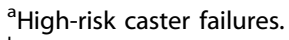

b Low-risk caster failures.

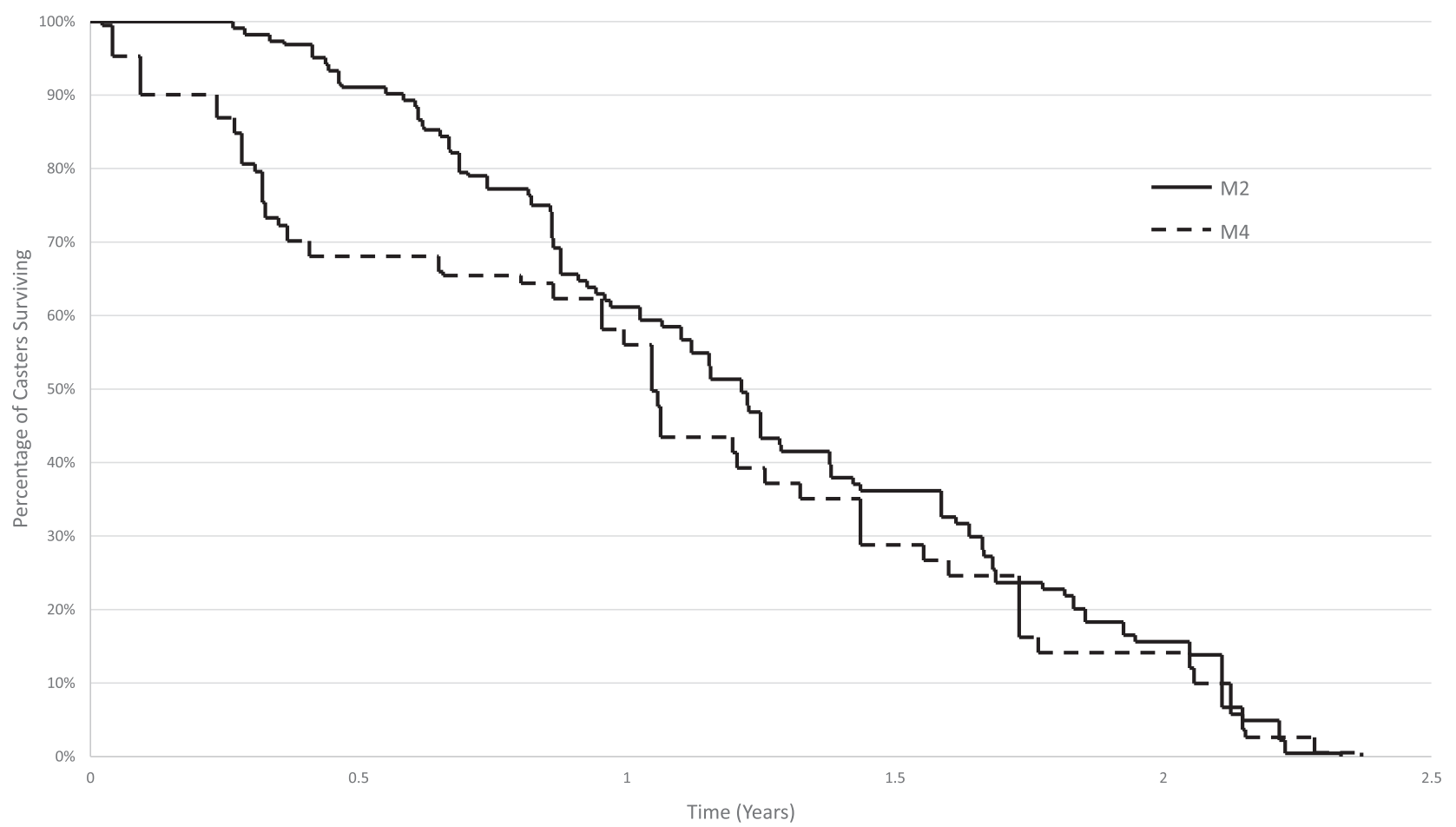

Fig. 1 Kaplan-Meier survival curves for high-risk M2 and M4 Group 3 power wheelchair caster failures.

users. The regression analysis for manual wheelchairs in Fig. 2 showed that service repairs reduced failure occurrences significantly. This result supports findings from previous wheelchair maintenance studies and highlights the need for preventative maintenance practices and training, especially for the vulnerable users of tilt-inspace wheelchairs $[4,13,14,24]$. Users should partake in maintenance training programs and educate themselves on using validated maintenance tools [21] to mitigate the risk of breakdowns and consequences. Some service repairs may not be performed owing to the complexity and the lack of tools and capabilities. In such cases, providers shall be incentivized by insurance for carrying out the repairs in collaboration with users and caregivers, perhaps remotely using telehealth approaches.

As per this study, casters incurred rapid fatigue, which made them prone to low- to high-risk failures as early as within a year of use. The rate of fatigue can subside, and early failures can be prohibited if 


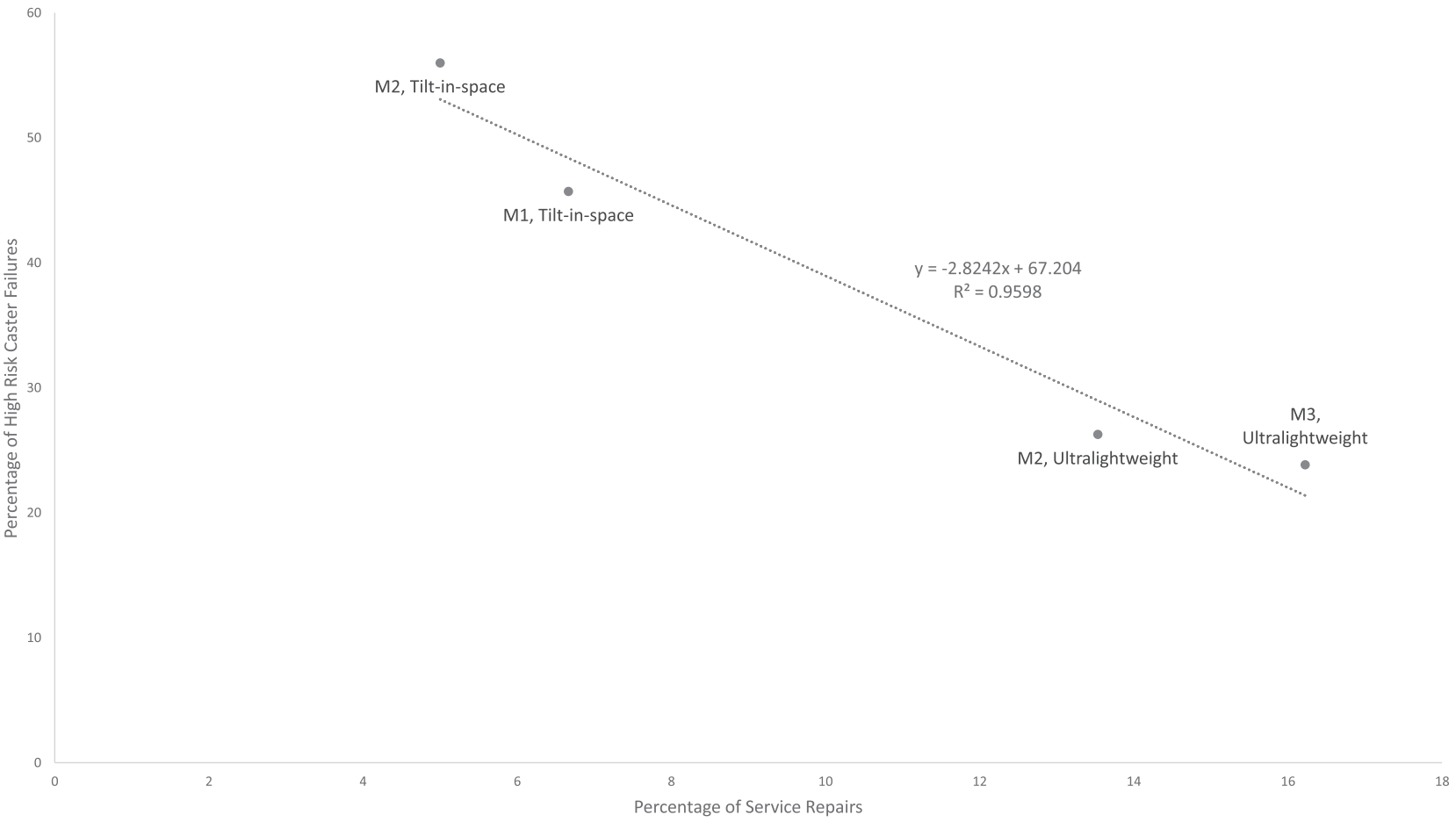

Fig. 2 Correlating manual wheelchair caster high-risk failures with service repairs. ${ }^{a}$ Wheelchair manufacturers (M\#) and the tilt-in-space and ultralightweight wheelchair models are included in the data callouts.

casters do not suffer from road impacts or misuse. Wheelchair skills training [25] enables users, depending on their abilities, to navigate architectural barriers and inaccessible outdoors. For instance, some trained users can wheelie over road bumps and curbs, thus avoiding caster shocks and fatigue. User education on skills and maintenance can prolong the survival of casters and wheelchair parts and prevent failures.

This study demonstrates the significant variability in caster performance across manufacturers and models. This finding should be of particular interest to clinicians, buyers, and insurance-the stakeholders in product selection. It is recommended that stakeholders seek information on standardized testing of products and product performances through reports, publications, and manufacturer specification sheets before selecting a wheelchair and/or wheelchair parts. At the same time, follow-up procedures at regular intervals are essential to understand and possibly document the reliability of prescribed wheelchairs.

One of the highlights of this study is the contrast in survival rates of the same caster models from two manufacturers M2 and M4. Such discrepancies are unwarranted as similar models from different manufacturers are procured at the same price cap, typically in developed countries. This rate increases with increasing group number of power wheelchairs. Group 3 and 4 power wheelchair casters experienced $21 \%$ more high-risk failures than Group 2 casters indicating that quality for caster used outdoors is inversely related to product cost. This raises a concern about cost reduction engineering practiced by some manufacturers in the wheelchair industry. Specifically, in the United States, this may result from the policies implemented by the Food and Drug Administration and CMS. Standardized wheelchair testing by independent testing laboratories remains a recommendation and not a requirement for all wheelchairs. Power wheelchair devices are durability tested by manufacturers as per CMS. These practices introduce a risk for bias. Enacting minimum standards as requirements for approval and public disclosure of related materials can ensure that appropriate quality matching the procurement cost is maintained by manufacturers.
This secondary data analysis study uses a larger dataset of wheelchair caster failures reported in the WRR to signify implications for stakeholders involved in wheelchair provision. It is anticipated that stakeholders identify key takeaways on wheelchair quality improvement and preventative maintenance and promote related processes and standards in their practices. On a broader level, regulatory agencies and federal insurance programs can take relevant steps towards informing policy and product procurement based on study outcomes.

\section{Study limitations}

The WRR data lacks data on wheelchair setup, provision, user training, user- or caregiver-led maintenance, user demographic characteristics, technician training, and use conditions that may influence failure type and frequency. Wheelchairs in use that did not encounter caster failures are not included in WRR and were not a part of the data analysis. However, a 45-63\% failure rate within 6-months of wheelchair use found in previous studies $[4,6,7]$ can be a suitable reference.

\section{Future work}

The future work includes laboratory testing of caster designs reported in the WRR. Comparing the testing results to failure findings reported in this study and communicating design changes to manufacturers is necessary to improve quality and design. Additionally, as the WRR data grows and more failure timepoints and purchase dates become available, it will be possible to reliably compute time to failure for multiple models and wheelchair parts, and inform the frequency of preventative maintenance events.

\section{CONCLUSION}

Wheelchair caster failures put wheelchair users at risk for multiple consequences, leading to decreased self-perceived health and quality of life. In this study, users who use wheelchair products that provide complex rehabilitation care and greater seating and positioning 
support were found to experience a greater number of high-risk caster failures. Service repairs are associated with a significant reduction in high-risk caster failures. Improvements in the quality and maintenance of wheelchair products are needed to mitigate the risk of failures and consequences suffered by wheelchair users.

\section{Data archiving}

The data analyzed in this study comes from the WRR hosted at the University of Pittsburgh [19]. The registry may be made available for public access following the completion of the grant.

\section{REFERENCES}

1. National Spinal Cord Injury Statistical Center. 2019 Annual Report for the Spinal Cord Injury Model Systems [Internet]. Birmingham, Alabama; 2019 https://www.nscisc.uab.edu.

2. Brault M. Americans With Disabilities: 2010 [Internet]. U.S. Census Bureau. 2012. https://www.census.gov/prod/2012pubs/p70-131.pdf

3. Wang H, Liu H-Y, Pearlman J, Cooper RRA, Jefferds A, Connor S, et al. Relationship between wheelchair durability and wheelchair type and years of test. Disabil Rehabil Assist Technol. 2010;5:318-22.

4. Toro M, Worobey L, Boninger ML, Cooper RA, Pearlman J. Type and frequency of reported wheelchair repairs and related adverse consequences among people with spinal cord injury. Arch Phys Med Rehabil. 2016;97:1753-60.

5. Mhatre A, Martin D, McCambridge M, Reese N, Sullivan M, Schoendorfer D, et al. Developing product quality standards for wheelchairs used in less-resourced environments. Afr J Disabil. 2017;6:15. pages

6. McClure LA, Boninger ML, Oyster ML, Williams S, Houlihan B, Lieberman JA, et al. Wheelchair repairs, breakdown, and adverse consequences for people with traumatic spinal cord injury. Arch Phys Med Rehabil [Internet]. 2009;90:2034-8. http://www.sciencedirect.com/ science/article/pii/S0003999309007151 [cited 2015 Jun 14]Available from

7. Worobey L, Oyster M, Nemunaitis G, Cooper R, Boninger ML. Increases in wheelchair breakdowns, repairs, and adverse consequences for people with traumatic spinal cord injury. Am J Phys Med Rehabil [Internet] 2012;91:463-9. http://www.ncbi.nlm.nih. gov/pubmed/22549473 [cited 2015 Jul 28]Available from

8. Gaal RP, Rebholtz N, Hotchkiss RD, Pfaelzer PF. Wheelchair rider injuries: causes and consequences for wheelchair design and selection. J Rehabil Res Dev 1997;34:58-71.

9. Kirby RL, Ackroyd-Stolarz SA, Brown MG, Kirkland SA, MacLeod DA. Wheelchair-related accidents caused by tips and falls among noninstitutionalized users of manually propelled wheelchairs in Nova Scotia. Am J Phys Med Rehabil 1994;73:319-30.

10. Veterans Health Administration. Veterans Health Administration Audit of the Timeliness of VISN 7 Power Wheelchair and Scooter Repairs [Internet]. 2018. www.va.gov/oig/hotline

11. Hogaboom NS, Worobey LA, Houlihan BV, Heinemann AW, and Boninger ML. Wheelchair breakdowns are associated with pain, pressure injuries, rehospitalization, and self-perceived health in full-time wheelchair users with spinal cord injury. Arch Phys Med Rehabil. 2018.

12. Fitzgerald SG, Collins DM, Cooper RA, Tolerico M, Kelleher A, Hunt $P$, et al. Issues in maintenance and repairs of wheelchairs: a pilot study. J Rehabil Res Dev 2005;42(Jan):853-62.

13. Hansen R, Tresse S, Gunnarsson RK. Fewer accidents and better maintenance with active wheelchair check-ups: a randomized controlled clinical trial. Clin Rehabil 2004;18:631-9.

14. Chen W-Y, Jang $Y$, Wang J-D, Huang W-N, Chang C-C, Mao H-F, et al. Wheelchairrelated accidents: relationship with wheelchair-using behavior in active community wheelchair users. Arch Phys Med Rehabil [Internet] 2011;92:892-8. https://doi.org/10.1016/j.apmr.2011.01.008. 1Available from

15. Centers for Medicare \& Medicaid Services. DMEPOS Competitive Bidding - Home CMS [Internet]. 2019. https://www.cms.gov/Medicare/Medicare-Fee-for-ServicePayment/DMEPOSCompetitiveBid/index

16. Medicare Interactive. DME repairs and maintenance - Medicare Interactive [Internet]. 2020. https://www.medicareinteractive.org/get-answers/medicarecovered-services/durable-medical-equipment-dme/dme-repairs-andmaintenance

17. Mhatre A, Reese N, and Pearlman J. Design and evaluation of a laboratory-based wheelchair castor testing protocol using community data. PLoS One. 2020. https://doi.org/10.1371/journal.pone.0226621

18. Mhatre A, Lachell S, and Pearlman J Development, reliability, and piloting of a wheelchair caster failure inspection tool (C-FIT). Disabil Rehabil Assist Technol. 2019 Feb;1-10.

19. James $A$, Igede $P$, Mhatre $A$, Brienza D, Pearlman J, Karg $P$, et al. Development of a Wheelchair Repair Registry. Arch Phys Med Rehabil. 2021; https://doi.org/ 10.1016/j.apmr.2021.02.023

20. Centers for Medicare \& Medicaid Services. Details for title: 2020|CMS. 2020. https://www.cms.gov/Medicare/Coding/HCPCSReleaseCodeSets/Alpha-NumericHCPCS-Items/2020-Alpha-Numeric-HCPCS-File
21. Toro Hernandez ML. Development, Implementation, and Dissemination of a Wheelchair Maintenance Training Program. University of Pittsburgh. 2016.

22. Rispin K, Riseling K, Wee J. A longitudinal study assessing the maintenance condition of cadres of four types of wheelchairs provided in low-resource areas. Disabil Rehabil Assist Technol 2018;13:146-56.

23. International Organization for Standardization. ISO/DIS 7176-32 - WheelchairPart 32: Standard Practice for Wheelchair Castor Durability Testing. 2021. https:// www.iso.org/standard/77566.html

24. Toro ML, Bird E, Oyster M, Worobey L, Lain M, Bucior S, et al. Development of a wheelchair maintenance training programme and questionnaire for clinicians and wheelchair users. Disabil Rehabil Assist Technol. 2017;12:843-51.

25. MacPhee AH, Kirby RL, Coolen AL, Smith C, MacLeod DA, Dupuis DJ. Wheelchair skills training program: a randomized clinical trial of wheelchair users undergoing initial rehabilitation. Arch Phys Med Rehabil. 2004;85:41-50.

\section{ACKNOWLEDGEMENTS}

The authors would like to sincerely thank the Rehabilitation Engineering Research Center members at the University of Pittsburgh and the Van G. Miller Group for their support to this study.

\section{AUTHOR CONTRIBUTIONS}

AM conceptualized the study, led the data analysis/interpretation, drafted the article and synthesized the findings. BK and JF were responsible for data cleaning, plotting and statistical analysis. JP and MS reviewed the article. JP secured funding for this study.

\section{FUNDING}

This study was funded under a grant from the National Institute on Disability, Independent Living, and Rehabilitation Research (NIDILRR grant number 90REGE0001-01-00). NIDILRR is a Center within the Administration for Community Living (ACL), Department of Health and Human Services (HHS). The contents of this dissertation do not necessarily represent the policy of NIDILRR, ACL, or HHS, and you should not assume endorsement by the Federal Government.

\section{COMPETING INTERESTS}

The authors declare no competing interests.

\section{STATEMENT OF ETHICS}

A data sharing agreement has been established between the University of Pittsburgh and VGM/U.S. Rehab for transfer and analysis of wheelchair repair data in the WRR. No Institutional Review Board approval was needed as data shared was de-identified and the study was not considered as human subjects research.

\section{ADDITIONAL INFORMATION}

Correspondence and requests for materials should be addressed to A.M.

Reprints and permission information is available at http://www.nature.com/ reprints

Publisher's note Springer Nature remains neutral with regard to jurisdictional claims in published maps and institutional affiliations.

\footnotetext{
Attribution 4.0 International License, which permits use, sharing, adaptation, distribution and reproduction in any medium or format, as long as you give appropriate credit to the original author(s) and the source, provide a link to the Creative Commons license, and indicate if changes were made. The images or other third party material in this article are included in the article's Creative Commons license, unless indicated otherwise in a credit line to the material. If material is not included in the article's Creative Commons license and your intended use is not permitted by statutory regulation or exceeds the permitted use, you will need to obtain permission directly from the copyright holder. To view a copy of this license, visit http://creativecommons. org/licenses/by/4.0/.
}

Open Access This article is licensed under a Creative Commons

(c) The Author(s) 2021 